\title{
FABIANISME ET COMMUNISME: LES WEBB ET L'UNION SOVIÉTIQUE
}

Sidney et Beatrice Webb sont incontestablement deux des personnalités les plus importantes et les plus représentatives du socialisme contemporain en Grande-Bretagne. Et si celui-ci traversait aujourd'hui une crise susceptible de le transformer, il n'en resterait pas moins marqué par les courants qui ont présidé à son premier envol.

Le fabianisme y figure en première place, et doit ses traits déterminants et son influence à l'impulsion quelui conférèrent Sidney etBeatrice Webb. On a dès lors pu dire que «la version que les Webb donnèrent du Socialisme... devint celle qu'adopta la Grande-Bretagne». ${ }^{1}$ Faut-il préciser que cette version s'inscrit dans le courant le plus nettement réformiste du socialisme européen? Il est significatif que, lors de la rupture du mouvement socialiste international, à la suite de la première guerre mondiale et de la Révolution bolchévique, lorsque se reconstituèrent, face à la IIIe Internationale communiste, et à côté de l'éphémère «IIe Internationale et Demie,» conciliatrice et velléitaire dans son désir de réformes comme dans ses aspirations révolutionnaires, les débris d'une IIe Internationale essentiellement réformiste, ce furent les socialistes anglais qui en constituèrent le noyau. Il est significatif aussi que lorsque se réunit un des premiers congrès de cette Ile Internationale reconstituée, à Genève au cours de l'été 1920, ce fut à Sidney Webb que l'on confia la rédaction de la résolution sur le «Système politique du socialisme».

Sans sa préoccupation fondamentale pour des études sociales, voire administratives, concrètes, sans sa méfiance instinctive à l'égard des grands principes idéologiques, Sidney Webb - inséparable d'ailleurs de Beatrice - eût été, avec Karl Kautsky, le principal idéologue du socialisme anti-communiste, démocratique et réformiste, et dans le cas des Webb, plus réformiste encore que démocratique.

Or, l'œuvre monumentale de ces éminents sociologues est couronnée

1 R. H. Tawney, The Attack and other papers. The Webbs and their work, Londres s.d., p. 4 I. 
par un livre, le dernier qu'ils publièrent, qui est comme une découverte, une conversion ou un reniement, une rupture apparente en tous cas avec un passé d'action et de recherches placé sous le signe du fabianisme réformiste. En novembre 1935 , paraissait la première édition de «Soviet Communism: a New Civilisation?» qui allait connaître une seconde édition en 1937 , une troisième en 1944 et dont le titre allait être allégé de ce point d'interrogation qui révélait la provisoire prudence de ses auteurs. Cette parution rendait publique une transformation à première vue radicale des idées que professaient Sidney et Beatrice Webb. Cette dernière, dès septembre 193 I, avant donc son voyage en Union soviétique, notait dans son journal: «... sans aucun doute, nous (Sidney et Beatrice) sommes aux côtés de la Russie» ${ }^{1}$ donnant, comme il ressort du contexte, une portée très générale à cette affirmation, alors que, dans une lettre publique, elle considérait, en 1927 encore, la Révolution russe comme «le plus grand malheur de l'histoire du mouvement ouvrier»! 2

Cette étude se propose de marquer les étapes de cette surprenante «conversion», l'expression étant utilisée avec des réserves que nous relèverons et justifierons en cours d'analyse. Nous aurons en effet à nous demander dans quelle mesure la découverte par les Webb de l'Union soviétique et des bienfaits de sa civilisation signifiait pour eux une rupture avec leur idéologie propre. En recherchant les causes de leur évolution, nous serons finalement amenés à poser le problème des relations entre le fabianisme et le communisme, ou plus exactement entre le fabianisme et la version du communisme à laquelle la Révolution russe donna naissance dans une société si peu préparée à tant d'égards à le recevoir.

$$
* * *
$$

L'étude de la pensée politique de Sidney et Beatrice Webb conduit nécessairement à analyser celle du fabianisme dont il est indispensable de dégager ici les grands traits.

$\mathrm{La}$ Société fabienne est née en $\mathrm{I} 884$ et le choix du nom qui lui fut donné est typique de la mentalité de ses fondateurs et de l'esprit qui l'anima pendant la plus grande partie de son histoire. Il évoque en effet le souvenir d'un général romain, Quintus Fabius «Cunctator», le Temporisateur. Sans doute, la Société fabienne, dans sa déclaration de base, affirmait-elle qu' «elle se compose de socialistes» ${ }^{3}$ et prenait-elle posi-

${ }^{1}$ Beatrice Webb's Diaries (1924-1932), edited by M. I. Cole, Londres, 1956, p. 287.

2 The Webbs and their work; B. Drake, The Webbs and Soviet Communism, Londres, I949, p. 22 I.

${ }^{3}$ Cité par G. D. H. Cole, A History of Socialist Thought; vol II, Marxism and Anarchism (1850-1890), Londres 1954, P. I25. 
tion en faveur de «l'établissement d'une société où sera assurée l'égalité des chances et où le pouvoir économique et les privilèges des individus et des classes seront abolis par l'appropriation collective et le contrôle démocratique des ressources économiques de la communauté». ${ }^{1}$ Mais si par les buts qu'elle fixait à son action, la société se rattachait à la grande famille socialiste, - elle envoyait d'ailleurs des délégués aux Congrès de la IIe Internationale -, elle se différenciait on ne peut plus nettement des partis ouvriers britanniques ou continentaux, tant par sa composition et son recrutement que par ses méthodes d'action.

En ce qui concerne sa composition sociale, la Société fabienne était essentiellement et même quasi-exclusivement une institution bourgeoise, ou plus précisément petite-bourgeoise. Au cours de ses premières années d'existence, elle ne compta qu'un seul ouvrier, W. L. Pease, auteur de la première brochure fabienne «Why Are the Many Poor?». ${ }^{2}$ $\mathrm{Ne}$ groupant, dix ans après sa fondation, que quelques centaines de membres, ${ }^{3}$ elle ne visait aucunement à rassembler les masses prolétariennes. C'était bien plus un cercle d'études qu'un parti politique.

Les méthodes par lesquelles elle espérait contribuer à l'instauration du socialisme n'avaient, en effet, rien en commun avec l'action politique, telle qu'on l'entend ordinairement: «elle opérait, comme des francstireurs intellectuels, sur le flanc des armées politiques régulières» ${ }^{4}$ et, selon le paragraphe final de sa déclaration de base, elle cherchait à réaliser ses buts «par la diffusion d'idées socialistes..., ainsi que par celle des connaissances relatives aux relations entre l'individu et la société, dans leur aspect économique, éthique et politique». ${ }^{5} \mathrm{Et}$ un des membres les plus influents de la société, George B. Shaw, confirmait son caractère d'institution quasi-académique dans une brochure datant de I 884: «Les fabiens sont associés afin de diffuser les idées suivantes... et d'en discuter les conséquences pratiques» ${ }^{6}$

Dans tout cela, il n'était naturellement pas question de lutte de classes, d'action politique autonome de la classe ouvrière où de revendications économiques du prolétariat. De telles méthodes n'avaient rien en commun avec l'esprit de la Société fabienne, soit qu'elle les condamnât, plus ou moins implicitement, soit qu'elle les jugeât étrangères à ses préoccupations.

${ }^{1}$ G. D. H. Cole, The Fabian Society, Past and Present (Fabian Tract no. 258), Londres, I 952 , P. I.

${ }^{2}$ G. D. H. Cole, A History of Socialist Thought, Vol. II, p. 107.

3 Pour ses effectifs à cette époque, voir ibid, p. 127.

4 R. H. Tawney, The Webbs in Perspective, Londres, 1953, p. 5.

5 Cité par G. D. H. Cole, A History of Socialist Thought, vol. II, p. 125.

- G. B. Shaw, A Manifesto (Fabian Tract no. 2), Londres, 1884. 
Il ne pouvait guère en être autrement étant donné les liens étroits qui rattachaient la plupart des fabiens au libéralisme, et plus particulièrement à son aile progressiste. Les thèses purement réformistes de Sidney Webb, dont la personnalité façonna très rapidement l'ensemble du mouvement, l'engageaient d'ailleurs à utiliser les instruments institutionnels et politiques existants plutôt que d'en vouloir créer de nouveaux. C'était là une de ses conceptions les plus fondamentales, une tactique fondée sur sa conception de l'évolution économique et politique de la société capitaliste: la «permeation», l'infiltration des idées socialistes dans l'esprit des hommes et des institutions jusqu'à les en imprégner complètement par un processus lent et presque imperceptible.

Le groupe politique qui semblait se prêter le mieux à une telle infiltration était l'aile radicale et progressiste du Parti libéral que le départ en 1886 de Joseph Chamberlain venait d'affaiblir, mais en même temps d'épurer. L'action des fabiens et leurs liaisons n'étaient toutefois pas exclusivement orientées dans cette direction: ils ne dédaignaient pas se lier ni à des libéraux de plus orthodoxe obédience, ni même à des conservateurs.

Dans ces conditions, il ne faut pas s'étonner outre mesure du fait que les fabiens aient manifesté longtemps une réticence prononcée à lier leur sort à celui des groupes politiques socialistes en voie de formation. Le développement, à partir de 1889 , d'un syndicalisme de type nouveau, le «New Unionism», à la suite d'une augmentation du chômage et d'une vague d'agitations sociales spectaculaires, contribua puissament à l'apparition de ces groupes socialistes, dont le plus important, l'«Independent Labour Party» (I.L.P.), fut fondé en 1893 par Keir Hardie. D'autre part, le refus de nombreux dirigeants libéraux d'admettre des candidats ouvriers aux élections générales, candidats dits «Lib-Lab» bénéficiant des voix ouvrières et de l'étiquette libérale, renforça la tendance qui aboutit finalement à l'autonomie politique du prolétariat anglais par la fondation du Labour Party.

Dès la création du I. L. P., George B. Shaw déclara, au nom de la Société fabienne, que celle-ci n'avait nullement l'intention d'adhérer au nouveau parti et comptait poursuivre sa tactique d'infiltration en direction de tous les groupes politiques existants. ${ }^{1}$

Pris individuellement, les fabiens, à Londres surtout, demeuraient au contraire fidèles au groupe progressiste que, sous la direction de Sidney Webb, ils formaient avec certains radicaux au London County Council, refusant de s'engager dans une action indépendante puisqu'aussi bien

${ }^{1}$ G. D. H. Cole, A History of Socialist Thought, vol. II, p. 152. 
«ils s'y comportaient bien plus en radicaux qu'en socialistes». ${ }^{1}$ En province, une telle attitude fut condamnée par de nombreux groupes fabiens qui se dissolvèrent pour rejoindre purement et simplement le I.L.P. Au sein même de la société, elle se heurtait régulièrement à de fortes oppositions. Ainsi, en $\mathrm{x}$ 906, alors que le Parti Travailliste venait de se fonder, ${ }^{2}$ les tentatives pour y faire adhérer la Société fabienne échouèrent. En I9Is encore, la majorité des membres refusa d'éliminer les fabiens adhérant au Parti Libéral. C'est en I 919 seulement, alors que la Société fabienne elle-même ressentait les effets de la fièvre révolutionnaire qui balayait l'Europe, qu'elle consentit à modifier ses statuts et à adhérer formellement au Parti Travailliste.

Ce n'est donc pas uniquement par ses tendances anti-marxistes que le fabianisme se séparait du socialisme. S'il en partageait les buts lointains, il s'en distinguait foncièrement dans l'action pratique et quotidienne. Une telle divergence n'était naturellement pas fortuite. Elle ne tenait pas seulement à des questions de tactique, mais à des considérations idéologiques profondes que la théorie webbienne de l'évolution sociale exprime parfaitement. ${ }^{3}$

$$
* * *
$$

En qualifiant de «réformiste» la conception webbienne de l'évolution économique et politique, on n'en rend compte que très imparfaitement. Il faut en effet apercevoir tout d'abord ce que le terme de réformisme contient d'ambiguïté. On oppose généralement la «tendance réformiste» à la «tendance révolutionnaire», comme si elles formaient deux tactiques entièrement distinctes de la lutte sociale et comme si elles s'excluaient mutuellement. Or, des partis tévolutionnaires ont pu se proclamer réformistes et, inversément, des partis réformistes se déclarer révolutionnaires, sans que de telles prétentions dussent nécessairement être taxées de camouflage ou de démagogie. La lutte pour des réformes partielles dans le cadre d'un régime que l'on combat peut à la fois augmenter la combativité des masses militantes et révéler les limitations de ce régime. Il y a donc un réformisme révolutionnaire, comme il y a une réformisme anti-révolutionnaire, pour qui l'évolution progressive d'une société suffit à en assurer la transformation harmonieuse et complète.

Ce n'est pas assez de dire que les Webb appartiennent à ce dernier courant et ce n'est pas tant de réformisme qu'il faut parler dans leur cas

1 Ibid., p. 12 r.

2 Il naquit de la transformation du "Labour Representation Committee", fondé en 1900 et représentant au Parlement des groupes ouvriers et socialistes autonomes.

${ }^{3}$ G. D. H. Cole, A History of Socialist Thought, vol. II, Pp. I26-7; B. Webb's Diaries (1912-1924); ed. by M. I. Cole, Londres, 1952, p. 38. 
que de gradualisme, avec ce que cette expression suggère quant au rythme de l'évolution sociale. Ce gradualisme se fonde sur une interprétation de l'histoire qui se rapproche à certains égards du marxisme, mais qui en diverge aussi sur des points fondamentaux.

Pour Sidney et Beatrice Webb, comme pour Marx, le moteur de l'histoire réside dans les phénomènes économiques, de telle sorte que, pour eux, les principaux artisans de la démocratie bourgeoise que connaît l'Angleterre ne sont pas les révolutionnaires de 1649 ou de 1689 , mais les Watt et les Arkwright à qui l'on doit la révolution industrielle. ${ }^{1}$ D'autre part, et surtout, le capitalisme entraîne une concentration industrielle et financière qui ouvre la voie à la socialisation des moyens de production, de distribution et d'échange. Ainsi, le socialisme se trouve dans le sens de l'histoire; il s'y inscrit comme une phase nécessaire que la réalité présente annonce déjà, au sein même du monde capitaliste.

Dans l'essai qu'il écrivit en I 887 pour la Société fabienne, «Facts for Socialism», Sidney Webb présente le socialisme comme un régime réalisant pleinement des tendances existant dans le cadre du capitalisme et s'y épanouissant progressivement. ${ }^{2}$ Cette conception s'accusera plus tard et sera élaborée de manière plus explicite. Ainsi, dans le chapitre qu'il écrivit pour les «Fabian Essays on Socialism», S. Webb déclarait deux ans plus tard: «Les principes du socialisme contemporain ne sont que l'affirmation consciente et ouverte de conceptions d'organisation sociale qui ont, pour une large part, déjà été inconsciemment adoptées. L'histoire économique de ce siècle offre un bilan presque continu des progrès du socialisme». ${ }^{3}$

Un point de vue aussi optimiste s'exprimait fréquemment sous la plume de Sidney Webb à cette époque de sa carrière. En 1 894, il estimait que «le jeune politicien, qui, il y a vingt ans encore, était un individualiste convaincu, est aujourd'hui un collectiviste empiriste.... Il attend de l'avenir une économie toujours plus collectivisée». ${ }^{4}$

Dès lors, il va de soi que la révolution ne peut être qu'un bouleversement inutile puisque tout semble si heureusement disposé à préparer le triomphe progressif, graduel jusqu'à être imperceptible, du socialisme. Certain jour, les plus perspicaces des citoyens découvriront que, depuis quelques années déjà, le collectivisme a définitivement supplanté l'entreprise privée, sans que personne ne s'en soit aperçu.

\footnotetext{
${ }^{1}$ Fabian Essays on Socialism, S. Webb, The Historic Basis of Socialism, Londres, 1889 , p. 4I.

${ }^{2}$ G. D. H. Cole, A History of Socialist Thought, vol. II, p. 109.

${ }^{3} \mathrm{~S}$. Webb, Fabian Essays on Socialism, p. 3 I.

${ }^{4}$ S. Webb, Socialism, True and False (Fabian Tract no. 5 I), Londres, 1894, reédité en 1907, p. 8.
} 
Le «catastrophisme marxiste» ne se concilie absolument pas avec d'aussi linéaires perspectives. Et, en effet, nul socialiste ne dénonça plus que Sidney et Beatrice Webb l'inutilité sanglante des révolutions. Fondamentalement, ils croyaient et souhaitaient que la socialisation de l'industrie se réalise sans qu'il faille bouleverser l'appareil économique, politique et social de la société. ${ }^{1}$

Pour eux, «la transformation soudaine et simultanée d'une société individualiste en société collectiviste n'est ni possible, ni concevable». ${ }^{2}$ Toute révolution apparaît dès lors comme une entreprise criminelle: évoquant une conférence qu'elle venait de donner, Beatrice Webb écrivait dans son Journal, à la date du I4 novembre I9 I 5 , que «l'Esprit de révolte qui recourt à la violence....... (est) de la même nature que l'Esprit de la guerre. La violence révolutionnaire, tout comme les guerres internationales, sont la manifestation d'un désir d'imposer à autrui sa volonté». ${ }^{3}$ La révolution, inutile «car l'urne électorale a rendu les barricades désuètes» ${ }^{4}$ est donc aussi immorale que la guerre. Même la Révolution française de 1789 n'a pas trouvé grâce aux yeux des Webb, puisque, à les en croire, "elle a retardé d'un siècle les progrès de la démocratie politique en Angleterre». ${ }^{5}$

S'ils étaient moins sévères pour la lutte des classes, ils ne la repoussaient pas moins formellement parce qu'elle constitue selon eux «un sabotage et un gaspillage». ${ }^{6}$ En I 923 encore, il ne voyaient de remède à la crise du monde occidental qu'ils identifiaient à celle du capitalisme, que dans une meilleure compréhension de la part des ouvriers et des patrons de leurs véritables intérêts. Telle était la portée de l'appel par lequel ils terminaient leur analyse de la «Décadence de la Civilisation Capitaliste».?

Ayant repoussé la révolution et la guerre des classes comme instruments d'édification du socialisme, les Webb n'optèrent même pas pour la méthode plus orthodoxe des luttes électorales. Si on examine leur apport fondamentalà l'histoire politique de la Grande-Bretagne contemporaine, on aperçoit qu'il consiste principalement en une immense œuvre de description et d'analyse d'institutions sociales et économiques et,

1 S. Webb, The Root of Labour Unrest (Fabian Tract no. 196), Londres, 1920, p. 15.

${ }^{2}$ S. Webb, Socialism, True and False, p. 3. Voir aussi: S. and B. Webb, A Constitution for the Socialist Commonwealth of Great-Britain, Londres, 1920, P. 104-5.

${ }^{3}$ Beatrice Webb's Diaries (191 2-1924), p. $4^{8}$.

${ }^{4} \mathrm{~S}$. and B. Webb, What Syndicalism means, Londres, I91 2, p. 139.

${ }^{5}$ M. I. Cole, Beatrice Webb, Londres, I 945, p. 166 où l'auteur cite une lettre de Beatrice Webb.

'S. and B. Webb, The Decay of Capitalist Civilisation, Londres, 1923, p. 164.

7 Ibid., p. 177 . 
sur le plan pratique, dans la mise en œuvre constante et systématique de leur influence personnelle au service de leurs idées. Nous avons déjà défini la tactique d'infiltration («permeation») dont ils furent les principaux protagonistes et qu'ils pratiquèrent souvent avec bonheur. Il s'agissait, avons-nous dit, d'imprégner d'idées - plutôt que d'idéaux socialistes des hommes et des institutions jugés perméables à une telle pénétration. A cette fin, les Webb faisaient feu de tout bois et si l'on ne craignait d'user d'un terme plus péjoratif que celui de "permeation», on dirait qu'ils n'hésitaient pas toujours à recourir à l'intrigue. Car, si sur le plan des conceptions et des œuvres théoriques, l'infiltration se faisait au moyen d'une abondante production littéraire et scientifique et d'un nombre incalculable de conférences, au niveau des réalités quotidiennes, elle prenait volontiers la forme de réceptions et de dîners où tout l'art de la maîtresse de maison consistait à placer ses invités de marque de telle façon qu'ils fussent disponibles pour le travail d'endoctrinement auquel on comptait les soumettre. C'est Beatrice Webb elle-même qui raconte comment, ayant convié à dîner Arthur James Balfour, le célèbre homme d'État conservateur, elle entreprit de «l'amuser et de l'intéresser, de saisir toute occasion pour lui insuffler la bonne parole ainsi que les données concernant la situation de l'instruction publique à Londres». ${ }^{1}$

De leur manière d'appliquer la tactique de «permeation», on a pu écrire que «tout au long de leur longue et étonnante carrière, ils ne cessèrent de tirer les ficelles et de tisser leur toile de façon à faire passer un de leurs projets de loi.... ou de 'conditionner' l'esprit de ceux qui avaient le pouvoir». ${ }^{2}$

Leurs efforts étaient souvent couronnés de succès et des ministres conservateurs, tels que Roseberry et Balfour, sans attendre que les Webb les entreprennent, allaient eux-mêmes au devant de leurs conseils. Ainsi, la loi sur l'enseignement que fit voter Balfour en 1903, et qui, soit dit en passant, accordait des privilèges importants aux écoles confessionnelles dépendant de l'Eglise anglicane, lui fut directement inspirée par Sidney Webb. ${ }^{3}$

On constate ainsi que le «réformisme» des Webb empruntait pour se réaliser des voies que le plus modéré des sociaux-démocrates n'aurait pu approuver. La tribune du Parlement les intéressait moins que ses couloirs; les campagnes électorales les laissèrent longtemps indifférents

${ }^{1}$ Cité par G. B. Oxnam, Personalities in Social Reform. The Scholar as Social Reformer: Sidney and Beatrice Webb, New-York, s.d., p. 4I.

${ }^{2}$ L. Woolf, The Political Thought and the Webbs, dans: The Webbs and their Work, p. 257 .

${ }^{3}$ R. C. D. Ensor, Permeation, dans: The Webbs and their Work, p. 69. 
et si les masses ouvrières, finalement, devaient bénéficier de leurs efforts, elles n'entraient pas pour autant dans leurs jeux et leurs calculs. Elles n'apparaissaient que comme l'objet, mais en aucune manière comme le sujet d'une action politique revendicative.

En définitive, s'il fallait qualifier le socialisme de Sidney et Beatrice Webb tel qu'il se dégage de leurs grandes œuvres théoriques, de leurs déclarations et de leur action, on pourrait suggérer une forme nouvelle de la doctrine socialiste qui leur appartiendrait en propre. Il ne s'agirait ni d'un «socialisme réformiste» dont ils n'eurent évidemment jamais le monopole, ni d'un «socialisme académique» parce que leur action dépassa largement les limites de l'analyse sociologique et des travaux de cabinet, mais bien davantage d'un «socialisme institutionnel».

Etablissant dans «Our Partnership» le bilan de sa carrière et de celle de son mari, Beatrice Webb écrit qu'«en tant que chercheurs scientifiques, nous nous consacrâmes à l'étude des institutions sociales» ${ }^{1}$ et il est vrai que, grâce à eux, la connaissance des institutions sociales qu'ils étudièrent devint, pour la première fois, scientifique. ${ }^{2}$ Cet intérêt primordial accordé à des institutions socio-économiques, telles que les syndicats, les coopératives et les pouvoirs locaux, se comprend d'autant mieux que, pour eux, le progrès politique et social réalisé en Angleterre au cours du XIXème siècle est dû essentiellement au développement et à l'adaptation «d'institutions adéquates». ${ }^{3}$ Quant à la poursuite de ce progrès, il dépend, toujours selon eux, de l'ajustement de ces institutions aux nécessités nouvelles.

Dans «A Constitution for the Socialist Commonwealth of GreatBritain» les Webb, affirmant que la condition de l'homme est déterminée par l'environnement où il s'insère, précisent que «de tous les éléments qui composent cet environnement...., le plus important n'est ni le climat, ou les famines..., mais les institutions qu'engendre chaque société». Et ils concluent: «C’est précisément parce que le facteur déterminant du milieu est constitué par nos institutions sociales que l'avenir de notre civilisation dépend de nous-mêmes. Nous pouvons façonner nos institutions de telle façon qu'elles donnent naissance à la société conforme à nos désirs». ${ }^{4}$

Et, en effet, ils tentèrent avant tout de se rapprocher de la société socialiste à laquelle ils aspiraient en agissant sur les institutions les plus appropriées, selon eux, à réalisercette évolution: syndicats, coopératives, pouvoirs locaux. Ils s'intéressèrent moins aux hommes qu'aux fonc-

1 L. Woolf, op. cit., p. 252.

2 Ibid., p. 254.

${ }^{3}$ S. and B. Webb, What is Socialism? dans: The New Statesman, 6.9.1913.

4 S. and B. Webb, A Constitution for the Socialist Commonwealth of Great-Britain, p. 99. 
tions; à l'administration autant qu'à la politique et bien moins aux masses, en tout cas, qu'aux organisations qui les encadrent. Ce «socialisme institutionnel» s'adressait à une élite d'organisateurs, d'administrateurs et d'experts. C'est sa composante bureaucratique. Il prétendait s'édifier par la techerche sociologique davantage que par l'action d'une classe. C'est sa composante scientifique. Nous verrons que Bureaucratie et Science sont des constantes que l'on retrouve, à des degrés divers, dans toute l'œuvre des Webb. Elles interviennent, entre autres, dans l'explication de leur «conversion» au communisme soviétique.

$$
* * *
$$

On devine quels sentiments Sidney et Beatrice Webb vouèrent à la Révolution d'Octobre quand elle éclata dans une Europe décimée par la guerre. Ils ne pouvaient que la condamner et ils hésitèrent d'autant moins à le faire que le triomphe communiste et la Paix de BrestLitovsk qui en résulta déforçaient, à première vue du moins, le camp allié dans sa lutte contre les Empires d'Europe Centrale.

L'attitude des Webb, au cours de la première guerre mondiale, fut sans équivoque, encore qu'il faille distinguer ici celle de Beatrice et celle de Sidney. ${ }^{1}$ Ce dernier, sans appartenir au clan des socialistes anglais les plus chauvins où s'illustrait Hyndman, n'en avait pas moins assumé les exigences d'un patriotisme intransigeant. Sa femme, quant à elle, en paraissait plus détachée sans pour autant éprouver la moindre sympathie pour les socialistes pacifistes.

Ainsi donc, l'hostilité des Webb à la Révolution bolchévique se fonda, dès 1917 , dans le chef de Sidney surtout, sur des raisons stratégiques autant qu'idéologiques. C'est ce qui explique qu'avec une minorité de dirigeants travaillistes, Sidney Webb se prononça en 1918 en faveur d'une intervention militaire alliée pour écraser les communistes. ${ }^{2}$

Pour apprécier pleinement des dispositions aussi agressives, il faut noter que, dans leur très grande majorité, les chefs travaillistes de l'époque se gardèrent bien d'adopter une position aussi tranchée. Sans doute, des hommes comme Snowden et comme Ramsay MacDonald surtout, manifestèrent-ils pendant quelque temps envers les bolchéviks une sympathie qui s'expliquait exclusivement par leur pacifisme. ${ }^{3}$ Mais

\footnotetext{
1 Pour l'attitude des Webb à l'égard de la guerre et du patriotisme, voir Beatrice Webb's Diaries (191 2-1924), p. 26, p. 33, p. 46-7; ainsi que M. I. Cole, Préface à Beatrice Webb's Diaries (191 2-1924), p. 9.

S. R. Graubart, British Labour and the Russian Revolution, Cambridge (Mass.), 1956, p. IIO-II 2.

sour l'attitude de R. MacDonald, voir M. Liebman, Ramsay MacDonald et la Révolution russe dans: Revue de l'Institut de Sociologie, Bruxelles 1956, pp. $547-576$
} 
le leader du parti lui-même, Arthur Henderson, bien qu'ancien ministre du gouvernement Lloyd George et rallié à la politique de guerre du pays, n'hésitait pas pour autant à exprimer aux ouvriers russes sa solidarité et à approuver, en substance, la paix de Brest-Litovsk. ${ }^{1} \mathrm{La}$ position de Sidney Webb à l'égard des communistes n'avait donc rien de modéré, ni de «centriste.» Il se situait à l'extrême-droite du mouvement travailliste.

Dans les années qui suivirent la Révolution d'Octobre, cette hostilité, pour être moins avérée, ne se relâcha pas pour autant. Comme nous l'avons vu, les Webb n'hésitèrent pas un instant à adhérer à la IIe Internationale reconstituée où ils firent partie de la majorité foncièrement anti-communiste. Cette hostilité se passe de justification. Elle est inscrite dans la philosophie même des Webb, dès lors qu'on fait abstraction de certains de ses caractères secondaires, mais non pour autant négligeables, qui eurent tout loisir d'affleurer par la suite. Tout au plus, peut-on noter que cette inimitié à l'égard des communistes russes était d'autant plus profonde qu'aux yeux des Webb, leur révolution apparaissait comme le triomphe de l'anarcho-syndicalisme auquel ils avaient toujours voué une haine sans réserve. Dans «The Decay of Capitalist Civilisation», ils résument à ce propos une opinion qui se retrouve dans nombre de leurs écrits: «Lénine eut l'occasion dans la Russie de 1918 et de 1919 de réaliser une grande partie des principes de Bakounine». ${ }^{2}$

Caractérisant cet anti-communisme, Margaret Cole affirme qu'«il est aussi absolu que les membres les plus anti-communistes du Parti Travailliste et des Syndicats eussent pu le souhaiter». ${ }^{3}$

Et en effet, le bolchévisme est tantôt qualifié par Beatrice Webb d'«épidémie», ${ }^{4}$ tantôt d' «entreprise ténébreuse». ${ }^{5}$ L'État soviétique est parfois qualifié d' «État tyran», ${ }^{6}$ parfois dénoncé comme un «capitalisme d'Etat de caractère bureaucratique». ${ }^{7}$ Les méfaits du bolchévisme sont comparés aux pires excès du capitalisme lors de la croissance industrielle en Angleterre. ${ }^{8}$ Quant à la dictature du prolétariat, à en croire les Webb, «elle est certainement une dictature.. mais comme avant la Révolution, le prolétariat la subit». ${ }^{9}$

1 S. R. Graubart, op. cité, p. 5 r-55.

${ }^{2}$ S. and B. Webb, The Decay of Capitalist Civilisation, p. 77.

${ }^{3}$ M. I. Cole, Beatrice Webb, p. 166.

4 Beatrice Webb's Diaries (1912-1924), 29.7.1918, p. 229.

5 Ibid., 21.1.1918, p. 107.

B Ibid., 1.7.I921, p. 183 .

7 B. Webb, dans: The Fabian News, juin rgar.

${ }^{8} \mathrm{~S}$. and B. Webb, The Decay of Capitalist Civilisation, p. 77.

9 Ibid, p. I6r. 
Il se passera encore une dizaine d'années avant que l'épidémie bolchévique ne se muât en «civilisation soviétique». Entre-temps, les Webb conserveront longtemps encore leur animosité envers l'Union soviétique. Elle s'exprime tout au long du Journal de Beatrice que des remarques anti-soviétiques émaillent jusqu'en 1927 . Ses critiques - qui, notons-le, ne prennent la forme que d'observations incidentes sans s'intégrer dans une analyse quelque peu poussée des faits - tournent autour de deux thèmes: l'assimilation du communisme russe au fascisme italien et leur condamnation commune; ${ }^{1}$ l'application au

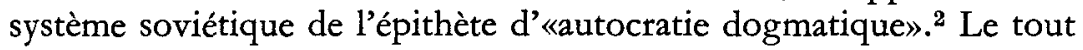
culminant dans cet anathème lancé publiquement par Beatrice et que nous citions au début de cette étude: «La révolution russe est le plus grand malheur de l'histoire du mouvement ouvrier».

$$
* * *
$$

Si la «conversion» des Webb ne fut révélée au grand public qu'en novembre 1935, lorsque parut leur monumental ouvrage «Soviet Communism: A New Civilisation?», tous ceux qui en connaissaient les auteurs la savaient acquise depuis plusieurs années déjà. Avant même leur arrivée en Russie, en mai 1932, le retournement avait été opéré et G.D.H. Cole, dans un essai qu'il publia pendant que les Webb sillonnaient l'Union soviétique, prédisait qu'ils en reviendraient enthousiasmés. $^{3}$

C'est qu'à partir d'une date que Margaret Cole situe aux environs du milieu de l'année $1930,{ }^{4}$ Beatrice Webb s'intéressait avec une curiosité de plus en plus passionnée à la Russie, lisant tous les livres, favorables et défavorables, traitant du Nouvel Ordre qui y avait été établi par la Révolution.

En 1930, elle vivait dans une retraite relative, éloignée des affaires publiques autant que le lui permettait la situation de Sidney. Celui-ci était depuis 1929 Secrétaire aux Colonies dans le deuxième Cabinet MacDonald. Et tandis que Sidney se débattait avec les problèmes que l'administration coloniale britannique rencontrait au Kenya et en $\mathrm{Pa}$ lestine, Beatrice éprouvait un sentiment de découragement toujours plus marqué devant le marasme de la politique anglaise et l'impuissance du Labour Party à y porter remède. Ce découragement confinait

1 Beatrice Webb's Diaries(1924-1932), 5.I 2.1925, p. 80; 30.6.1926, p. 10; 18.7.1927, p. 147 .

2 Ibid., 21.6.1925, p. 64; 30.6.1926, p. 106; 28.7.1927, p. 147 .

3 G. D. H. Cole, Persons and Prophets: The Webbs and the New Order, Londres, 1938 , pp. $321-332$.

4 M. I. Cole, Beatrice and Sidney Webb, Londres, 1955, p. 42. 
souvent au dégoût et le désarroi qu'il révélait chez une femme qui, jusqu'alors, s'etait entièrement consacrée aux affaires publiques de son pays, constitue un facteur psychologique important dans la découverte de l'U.R.S.S. par les Webb. On notera d'ailleurs que de Sidney et Beatrice, ce fut de toute évidence cette dernière qui entraîna son partenaire vers des horizons idéologiques auxquels il songeait moins encore qu'elle-même.

Le Journal de Beatrice Webb révèle abondamment ses croissantes inquiétudes morales et politiques. Moralement, elle se sentait toujours davantage frustrée dans un désir de pureté que l'âge semble avoir aiguisé. Dès 1925 , elle avouait ses troubles de conscience: «Le monde d'aujourd'hui offre beaucoup trop d'agréments aux membres du Parti Travailliste (elle visait certainement ses dirigeants bien plus que ses militants) qui en réalité appartiennent maintenant à la classe dirigeante». ${ }^{1}$ Le thème est abandonné pendant quelques années mais revient plusieurs fois sous sa plume en I 93 I, à l'époque critique où elle découvrit la Russie: «Tout le gouvernement [travailliste] et nous deux aussi, devons admettre que notre solidarité pour les chômeurs ne résiste pas aux effets du confort dans lequel nous vivons et de la liberté dont nous jouissons». ${ }^{2}$ Et encore: «Ce qui a fait la perte du Gouvernement travailliste, ce n'est pas seulement l'ignorance et l'absence de volonté,... mais aussi le fait que ses membres ont accepté de mener l'existence des classes riches et aristocratiques. Il est impossible à ceux qui vivent dans le faste et l'opulence au sein même d'une société misérable d'édifier un Etat égalitaire». ${ }^{3}$

A ces sentiments de frustration venait s'ajouter l'angoisse que lui inspirait la conviction de la décadence du monde occidental, et de son pays en particulier. Ce déclin de l'Angleterre est affirmé à maintes reprises dans son Journal, parfois sous forme de crainte, mais également comme une décevante constatation. ${ }^{4}$ Le sort du monde lui para1ssait devoir dépendre de la compétition entre les Etats-Unis capitalistes et la Russie communiste, la Grande-Bretagne elle-même ne faisant figure que de pion sur l'échiquier. ${ }^{5}$ En outre, elle avait depuis longtemps perdu toute confiance en ce Parti Travailliste dont elle avait contribué à modeler le visage. La déception qu'il lui causait s'exprimait par des remarques acerbes que ne tempéraient ni les nuances, ni l'indulgence. Dès avant $193 \mathrm{I}$, avant donc l'échec retentissant du

\footnotetext{
1 Beatrice Webb's Diaries (1924-1932), 12.2.1925, p. 56.

2 Ibid., 18.8.193 I. p. 275.

3 lbid, 28.ro.1931, p. 295.

4 Ibid., I8.5.1926, p. 99; 31.5.1926, p. 103;30.6.1926, p. 107; 29.11.1928, p. I86.

5 Ibid., 23.9.1931, p. 291.
} 
deuxième cabinet MacDonald, Beatrice Webb jugeait le Labour Party sans ressort et sans possibilité. Ses dirigeants appartiennent aux classes gouvernantes, «mais le Parti est incapable de gouverner».1 Leur présence au pouvoir «est une absurdité».2 Toujours à propos des leaders socialistes anglais, elle avait noté dès ig25 que «le Parti Travailliste est sans direction... (Parmi ses chefs) ceux qui ont du courage sont sans intelligence et ceux qui font preuve d'intelligence manquent de courage»; ${ }^{3}$ et que «la mauvaise situation du Parti est due à la distance qui sépare la classe ouvrière de ses chefs, installés dans leur conforts. 4

Cette critique ne se limitait pas au mouvement socialiste anglais: "Le mouvement ouvrier est tombé bien bas dans tous les pays capitalistes», écrivait Beatrice Webb en I93 I; «sur le plan des individus ainsi que comme force politique, il a cessé de compter, aussi bien aux Etats-Unis qu'en Europe et jusqu'en Australie et en Asie». ${ }^{5}$

Ainsi, face au capitalisme dont la crise économique et la situation sociale des Etats-Unis révélaient plus que jamais les imperfections et l'injustice, il ne restait plus qu'une puissance, celle du communisme russe. Telle était la conclusion plus ou moins nettement formulée à laquelle Beatrice avait abouti. Il lui restait à faire admettre ces vues pessimistes par Sidney et à étudier ce communisme soviétique, comme ils avaient analysé les institutions et la décadence de la société capitaliste.

Sidney Webb est longtemps demeuré confiant dans les possibilités du socialisme occidental et, plus généralement de la démocratie parlementaire. En 1923, il avait prononcé, lors de son élection comme Président du Parti Travailliste, une adresse demeurée fameuse où il avait déclaré une fois de plus que «de tous côtés, des parties de notre programme [socialiste] ont déjà été mises à exécution par les conseils municipaux, par les conseils de comté et par le gouvernement luimême... Toute la nation s'est imprégnée de socialisme sans même s'en rendre compte». ${ }^{6} \mathrm{La}$ théorie et la tactique de la "permeation» conservaient tous leurs droits.

Tandis que Beatrice lui faisait part de son scepticisme quant à l'avenir du socialisme occidental, Sidney tentait de la rassurer en réaffirmant que «nous glisserons vers un État égalitaire, comme nous avons glissé vers la démocratie... Les discours révolutionnaires

1 Ibid., 22.6.1925, p. 65; 5.4. 1927, p. 137 .

Ibid., 16.9.1925, p. 7 r.

${ }^{3}$ Ibid., 16.9.1925, p. 71 .

Ibid.,

Ibid., 25.1 2.1931, P. 296.

- Labour Party, Report of the 23d Annual Conference, Londres, 1923, p. 179. 
peuvent retarder une telle évolution, mais ne peuvent l'empêcher», ${ }^{1}$ sans pour autant parvenir à la convaincre. Beatrice elle-même notait qu' «il [Sidney] croit toujours que l'infiltration des autres partis par le fabianisme constitue le moyen le plus rapide pour établir un gouvernement vraiment socialiste». ${ }^{2}$ En 1926 , il «se moquait des craintes»de sa femme: «Même si la faillite de l'Europe devait se réaliser, il resterait toujours les Etats-Unis(dont) la puissance ne sera pas paralysée par nos conflits sociaux et nos atermoiments». ${ }^{3}$ Et jusqu'en 193 I, elle notait que Sidney croyait toujours dans ses théories de lente et imperceptible évolution vers le socialisme, tandis qu'elle-même se "prend à douter de l'inévitabilité du gradualisme et même de sa possibilité». ${ }^{4}$

Sans nous arrêter davantage sur cette divergence de vues qui se résorba rapidement et largement en faveur des thèses de Beatrice, indiquons cependant que Sidney était trop engagé dans l'action politique, notamment entre 1929 et 1931 , pendant qu'il exerçait ses fonctions de ministre, pour se livrer aux méditations et aux recherches qu'impliquait la remise en cause d'un anti-soviétisme considéré comme allant de soi.

Beatrice, au contraire, s'inquiétait et se documentait, soit par d'abondantes lectures, soit par des entretiens avec des partisans et des adversaires du communisme. En 1930, George B. Shaw, revenant de Russie, avait proclamé son enthousiasme, non sans influencer ses amis Sidney et surtout Beatrice. ${ }^{5}$ Les Webb, en outre, s'étaient pris d'amitié pour l'ambassadeur de l'Union soviétique à Londres et pour sa femme. Ils se fréquentaient assidument, échangeaient leurs opinions, les Webb recevant du diplomate plus qu'ils ne donnaient en retour. Tant et si bien qu'en juillet $193 \mathrm{I}$, Beatrice notait que l'ambassade russe «est le seul endroit où je me sente à l'aise». ${ }^{6}$

Sa «conversion» était déjà un fait acquis. Plusieurs autres remarques et annotations du Journal révèlent à quel point cette admiration fraîchement éclose pour l'Union soviétique avait été rapidement intégrée à la personnalité de Beatrice Webb, dont l'esprit était disponible pour de nouvelles allégeances.

En juin I930 déjà, comparant les deux grandes forces rivales, le capitalisme anglo-saxon et le communisme soviétique, elle écrivait que «tant pour leur foi et leur sincérité qu'en ce qui concerne le dévouement

\footnotetext{
1 Beatrice Webb's Diaries (1924-1932), 16.9.1925, p. 71.

2 Ibid.: 19.9.1925, p. 73.

3 Ibid., 18.5.1926, p. 99.

4 Ibid., r 2.2.1931, p. 269.

5 Ibid., préface de M. I. Cole, p. XVI.

B Ibid., r 3.7.1931, p. 275.
} 
au bien public... la classe dirigeante russe semble supérieure aux gouvernants anglo-saxons». ${ }^{1}$ En août de la même année, répliquant, en présence de l'ambassadeur soviétique, à Philip Snowden, Chancelier de l'Echiquier travailliste , qui se plaignait des ouvriers anglais toujours insatisfaits et revendiquant des salaires toujours plus élevés et des avantages sociaux toujours plus considérables, Beatrice Webb s'écriait: «Ah, Mr. Snowden, vous n'arriverez pas à faire travailler l'ouvrier anglais plus durement et pour un salaire moins élevé tant qu'il verra le patronat vivre dans le luxe. C'est l'égalité des revenus qui permet au gouvernement soviétique de demander et d'obtenir de la part de l'ouvrier russe une plus grande énergie au travail et davantage de sacrifices; les ouvriers russes savent qu'ils travaillent non pour les enfants des autres, mais pour leurs propres enfants».2

Finalement, elle déclarait au terme d'une évolution dont la rapidité offrait au gradualisme d'antan le démenti le plus cinglant: «Nous sommes aux côtés de la Russie». Et, en effet, Sidney, une fois débarrassé des responsabilités et des charges ministérielles, avait retrouvé le temps et la liberté d'esprit nécessaires pour partager les préoccupations de Beatrice d'abord, ses aspirations et sa foi ensuite.

En mai 1932, Sidney et Beatrice Webb s'embarquaient pour la Russie. Dès janvier de cette année, Beatrice reconnaissait qu'ils s'y rendaient animés d'un préjugé en faveur de son régime, ${ }^{3}$ encore qu'elle parût décidée à appliquer à l'étude des institutions soviétiques l'esprit de critique objective caractéristique de leur œuvre sociologique. ${ }^{4}$

$$
* * *
$$

Nous n'entreprendrons pas ici d'analyser dans leur totalité, et moins encore de résumer, les quelque douze cents pages d'un texte extrêmement dense que constitue le retentissant livre de Sidney et Beatrice Webb «Soviet Communism: A New Civilisation». Nous nous attacherons toutefois à en dégager le plus fidèlement possible les chapitres et les thèmes qui nous paraissent les plus significatifs, en distinguant l'aspect purement descriptif des prises de position personnelles pour insister davantage sur ces dernières.

La première partie de l'œuvre, qui dans les deux premières éditions en formait le premier volume, est essentiellement descriptive et, de manière fouillée, analyse les principales institutions soviétiques. Une

1 Tbid., 22.6.1930, p. 246.

2 Tbid., 3.8.1930, p. 249 .

Ibid., 4.1.1932, p. 298 ; 5.4.1932, 0. 305.

Ibid., 25.12.1931, p. 296. 
importance particulière est accordée aux syndicats et au Parti communiste.

En ce qui concerne les syndicats soviétiques, les auteurs rendent compte abondamment des controverses qui divisèrent le Parti dans les années 1920 . Les thèses de Tomski réclamant une relative autonomie des syndicats par rapport à l'État y sont largement exposées. ${ }^{1}$ On sait qu'elles furent mises en échec et que la volonté centralisatrice et étatiste de Staline l'emporta. Pourtant, dans leur description des rouages et des fonctions des syndicats, Sidney et Beatrice Webb n'explicitent nullement et ne commentent absolument pas cet «embrigadement» des institutions syndicales dans l'appareil étatique. La chose leur paraît au contraire naturelle et justifiée par la disparition du patronat et l'absence de tout antagonisme de classes en Russie: les délégués syndicaux en U.R.S.S. ne doivent pas affronter un "parti ennemi» et les conflits sociaux s'y résolvent donc à l'amiable. Sans doute, surgit-il parfois des «divergences de vue» entre les ouvriers et la direction de leur usine, mais la conciliation est assurée, de telle sorte que «la suspension du travail par la grève est pratiquement inconnue». ${ }^{2}$ La collaboration des syndicats à la réalisation du Plan est largement examinée et favorablement commentée, de même que la large participation des ouvriers à la vie syndicale et aux fonctions qu'elle implique. Dans l'ensemble et pour l'ensemble de ce chapitre, on trouve un examen approfondi et abondamment documenté, mais qui pèche par un simplisme et une acceptation tacite de données et de structures de base dont les liaisons avec l'institution étudiée sont mentionnées, mais sans qu'il en soit dégagé les conséquences pourtant fondamentales.

Le Parti communiste fait l'objet d'une étude plus minutieuse encore, tant en ce qui concerne son histoire que son organisation et que son esprit. Les Webb lui reconnaissent à la fois la vocation et la responsabilité de la direction des affaires publiques et analysent sa structure cônique: une base large et démocratique se restreignant à mesure qu'on approche du sommet autoritaire dont découlent les ordres, les directives et l'orientation politique. ${ }^{3}$ Les auteurs considèrent que l'existence et l'autorité du Parti sont «les caractéristiques dominantes du Communisme soviétique» et ne cachent pas que «la concentration des pouvoirs en un corps aussi discipliné a ses inconvénients»; d'autant plus que les congrès du Parti ont cessé d'être annuels et ne se tiennent plus que rarement et de manière irrégulière et que le Comité central qui, d'après

${ }^{1}$ B. and. S. Webb, Soviet Communism: A New Civilisation, ze ed., Londres, I944, pp. ×30-132.

2 Ibid., p. 575 et p. 143.

3 lbid., p. 320. 
les Statuts, devrait renseigner les organes du Parti sur ses travaux, ne se conforme pas à cette règle et entoure ses délibérations du secret le plus strict. ${ }^{1}$ Ces entorses graves au principe de la démocratie ne soulèvent de la part des auteurs aucun commentaire et n'empêchent pas Beatrice Webb d'écrire dans sa préface à la ze édition que «le Parti communiste... est démocratique dans sa structure interne puisqu'un congrès annuel y élit un Comité central qui, à son tour, désigne le Politbureau et les autres organes du Parti». Notons ici que cette préface developpe un point de vue plus favorable encore à l'U.R.S.S. que le livre lui-même. Il faut y voir le signe de l'évolution des opinions des Webb sur la Russie après la première édition de leur ouvrage; mais il ne faut pas non plus perdre de vue que cette préface fut écrite en février 1942, alors que l'Union soviétique, alliée aux Puissances occi-. dentales, subissait tout le poids de l'agression hitlérienne et jouissait, dans les milieux de gauche surtout, d'un regain extrême de popularité.

Dans l'ensemble, Sidney et Beatrice Webb font l'éloge du Parti communiste d'U.R.S.S. qu'ils présentent comme «l'instrument grâce auquel une élite politique, intellectuelle et législative a été donnée au peuple soviétique, élite qui le dirige par son dévouement désintéressé, sa formation supérieure et son sens des réalités ... et guide ses premiers pas incertains dans les voies de la liberté». ${ }^{2}$

Cet éloge se complète et se justifie par le portrait du «membre-type» du Parti communiste où sont dégagés les mobiles qui, selon les Webb, commandent son action et qui sont, en premier lieu, la foi dans la justesse de la cause et dans l'avenir du régime ainsi que le souci légitime d'exercer une influence et d'épanouir pleinement sa personnalité. Les purges, qui régulièrement éclairent les rangs du Parti, auraient pour principal, sinon pour exclusif, objet d'en éloigner les opportunistes et d'en conserver une pureté morale confinant au puritanisme. ${ }^{3}$

Ces appréciations flatteuses ne se comprennent qu'en tenant compte de l'espoir, assez vague, mais cependant certain, que Beatrice Webb nourrissait depuis longtemps de voir se créer en Occident un parti, très différent de l'image qu'offraient les mouvements socialistes, et qui aurait hérité des ordres religieux la foi, le désintéressement et la discipline. ${ }^{4}$

Cette analyse des structures et du rôle du Parti communiste soviétique est liée aux thèses que défendent les Webb concernant la démocratie en

1 Ibid., p. $908 .$, p. 281, p. 284.

B. Webb, Préface d̀ la ze éd. de Soviet Communism, p. XX.

3 Ibid., p. XXXIX.

- Soviet Communism, pp. 288-29o. 
Russie. Les auteurs de «Soviet Communism» s'emploient en effet à démontrer que le régime en vigueur en U.R.S.S. constitue une démocratie, en aucune façon une dictature. Ce n'est certes pas la partie la plus convaincante de leur cuvre. Plus que toute autre, elle souffre de l'inclusion dans des passages descriptifs ou analytiques de remarques idéologiques, souvent incidentes et parfois contradictoires car peu systématiques et insuffisamment rigoureuses.

Ainsi, Sidney et Beatrice Webb affirment, d'une part, que la dictature du prolétariat érigée par l'U.R.S.S. en système de gouvernement n'est en fait que la dictature du Parti, mais ils ajoutent tout aussitôt que «le contrôle que le Parti exerce sur l'administration ne se manifeste par aucune disposition contraignante pour le citoyen ordinaire...» ${ }^{1} \mathrm{En}$ outre, le Parti «ne peut par lui-même compléter ou changer les lois... Il ne peut qu'élaborer des directives... ne s'appliquant qu'à ses propres membres...; les membres du Parti, ainsi orientés dans leur action, ne peuvent agir que par persuasion». De telle sorte que «le terme de dictature ne convient certainement pas pour qualifier cette inlassable activité d'inspiration collective». ${ }^{2}$ On ne peut s'empêcher de penser que les auteurs eussent été mieux avisés s'ils avaient accumulé des arguments aussi convainquants non pour démentir, mais pour démontrer leur principale assertion.

Ils ne sont guère plus heureux dans les passages où ils traitent de l'autorité de Staline qu'ils n'ont garde de confondre avec une dictature personnelle. Et ils expliquent pourquoi : contrairement à ce qui se passe dans l'Allemagne nazie et dans l'Italie fasciste, la Constitution soviétique n'investit aucun individu d'un pouvoir dictatorial. De plus, la conduite de Staline prouverait qu'il n'est pas un dictateur et qu'il n'aspire d'ailleurs pas à la dictature. Il lui arriverait en effet - c'est lui-même qui le déclare - d'appliquer les instructions du Comité central du Parti. Tout le monde convient, au surplus, que Staline n'est pas assez vaniteux pour se croire infaillible. Enfin, l'histoire de la Russie soviétique et du Parti communiste prouverait qu'en des matières importantes, des décisions ont été prises sans la promptitude qui aurait trahi la marque indélibile de la dictature.

Tout cela permet à Sidney et à Beatrice Webb de conclure: «En résumé, le régime soviétique... a incarné... l'exact contraire d'une dictature. Il s'est révélé être un gouvernement exerçant son autorité par l'intermédiaire d'une série de comités»; bien mieux: «la structure

1 Beatrice Webb's Diaries (1924-1932); 28.10.1931, p. 295.

${ }^{2}$ Soviet Communism, p. 332. 
générale (des institutions soviétiques) montre un respect exagéré pour le principe des décisions collégiales...»1

Il n'y aurait donc pas en Russie de dictature d'un homme. Mais bien que, selon les Webb, Staline ne soit pas un dictateur, il n'en est pas moins vrai, et les auteurs ne font aucune difficulté pour l'admettre, qu'on entretient en Russie le culte du chef. Un tel culte, cependant, leur paraît justifié par le caractère arriéré des masses russes, «incapables de comprendre la philosophie nouvelle du Parti communiste». Certes, cette idolâtrie choque la sociologue britannique qui se rassure en décrétant que «le culte de Staline a, dans une large mesure, cessé d'exister dans la Russie d'aujourd'hui» et qu'en tous cas, cette «maladie infantile» disparaîtra à mesure que l'instruction se répandra dans les masses». ${ }^{2}$

Enfin, mettant le point final à un chapitre intitulé »Dictature ou Démocratie?», les auteurs expliquent qu' «il n'y a pas, en sociologie, de source d'erreurs plus fertile que de poser des problèmes, en termes tirés d'anciennes catégories, ou de définitions périmées», «l'aristocratie, l'oligarchie et la démocratie» leur paraissant précisément appartenir à cette terminologie désuète. ${ }^{3}$

L'argument aurait valu qu'on s'y arrête si les auteurs eux-mêmes avaient jugé utile de lui consacrer le développement qu'il semble mériter et si, surtout, ils n'avaient pas, une fois de plus, avoué le peu de crédit qu'ils lui accordent en affirmant que la Russie soviétique est une démocratie. Ils précisent même que c'est «une démocratie multiforme».4

Certes, ce n'est pas une démocratie libérale, un tel régime étant de toute évidence impossible en Russie. ${ }^{5}$ Mais l'égalité des races que le Gouvernement soviétique a substitué à l'oppression des minorités prouve la réalité de la démocratie soviétique; ${ }^{6}$ davantage encore: «Jugée en fonction de la constitution de r936, l'U.R.S.S. est la plus large et la plus égalitaire des démocraties du monde». ${ }^{7}$ Enfin, et de manière plus convaincante car moins formaliste, les Webb tirent argument de l'intervention active des masses soviétiques dans les affaires publiques, que ce soit sous forme de meetings où sont discutés le Plan quinquennal ou la Constitution, ou sous la forme de la participation bénévole de dizaines de milliers d'ouvriers à certaines fonctions syn-

1 Ibid., pp. 333, 334, 338, 336,337, 331 .

2 Préface de B. Webb. pp. XLIV, XLV.

a Soviet Communism, p. 347.

4 Ibid., p. 330.

5 Ibid., p. 245.

- Préface de B. Webb, p. XXII.

7 Ibid., p. XXI. 
dicales ou aux tâches qu'implique la gestion des affaires par les soviets locaux. ${ }^{1}$

Pour être complet, ajoutons que le système du parti unique est avalisé par les auteurs, et principalement par Beatrice qui renforce son argumentation par une critique véhémente des différents partis politiques occidentaux, mettant en cause le principe même de la multiplicité des partis. ${ }^{2}$

«Soviet Communism» eût été une cuvre partisane si les Webb n'avaient consacré un développement relativement important aux aspects négatifs de la société communiste et n'avaient traité tour à tour de la «terreur rouge» et, plus généralement, des excès du régime, ainsi que de ce qu'ils appellent la «maladie de l'orthodoxie». ${ }^{3}$

«Nous craignons qu'on ne puisse mettre en doute la réalité de la terreur rouge», ${ }^{4}$ écrivent-ils, récapitulant l'histoire de la Russie depuis la Révolution de février 1917. Mais cette terreur n'était-elle pas inévitable en période de révolution et, à fortiori, de guerre civile?

Les excès et les abus d'un pouvoir déjà installé retiennent plus longuement leur attention. Très objectivement, ils retracent l'histoire des organes de répression soviétiques, Tchéka, G.P.U., N.K.V.D., ne dissimulant rien de l'arbitraire auquel il leur est maintes fois arrivé de recourir avec les terribles conséquences qu'il a entraînées, ${ }^{5}$ mais s'efforçant également de situer les faits dans leur contexte historique, social et politique et rejetant la solution de facilité que constitue la seule invocation de la «tyrannie communiste» ou de la «cruauté asiatique».

Ainsi, les mesures d'épuration et de répression prises dans les années 1929-1930 contre l'intelligentsia russe et une partie des cadres techniques sont impartialement rapportées et leurs causes sérieusement analysées. ${ }^{6} \mathrm{Il}$ en va de même de la déportation des «koulaks». Et si les causes de cette mesure sont expliquées avec la volonté de comprendre plus qu'avec le désir de s'indigner et de condamner, ${ }^{7}$ Sidney et Beatrice Webb n'hésitent pas à constater que «la somme de souffrances humaines (ainsi causées) est au-delà de toute évaluation.» ${ }^{8}$

Par contre, la vague d'arrestations et de condamnations qui suivit l'assassinat de Kirov, en 1934, leur paraît plus difficilement explicable. D'autant moins explicable, en tous cas, que la responsabilité du gou-

${ }^{1}$ Soviet Communism, pp. 18, 38, 39, 906.

2 Préface de B. Webb, pp. XXIII, XXIX, XXXI.

3 Soviet Communism, pp. 801, 807 .

4 Ibid., p. 474.

5 Ibid., pp. $47^{2}, 47^{8}, 479$.

' lbid., pp. $457,458$.

7 Ibid., p. 47 I.

8 Ibid., p. 468. 
vernement soviétique et de Staline est établie dans ce cas, alors que pour la lutte contre l'intelligentsia, les auteurs s'étaient efforcés de dissocier la direction suprême de l'U.R.S.S. des organes d'exécution, tel que la G.P.U., qui parfois échappaient à son contrôle. Il teste néanmoins que la critique des autorités soviétiques en cette matière est empreinte de modération ${ }^{1}$ et que la persécution des trotskistes par exemple est complètement passée sous silence.

Les Webb rendent d'autre part hommage à certains aspects de l'œuvre de la G.P.U. qu'ils jugent «constructifs», tel que l'utilisation à des travaux servant au bien de la communauté de condamnés qui, dans certains cas du moins, loin de faire figure de bannis, retrouvent ainsi un rôle social utile et le sens de leur dignité. ${ }^{2}$

Enfin, l'orthodoxie dogmatique et quasi-scolastique de la "philosophie communiste» est mise en lumière et critiquée par les auteurs, sans sévérité excessive, mais sans complaisance. Ils en citent de nombreux exemples et regrettent les effets débilitants qu'elle ne peut manquer d'avoir sur les intellectuels et sur la recherche scientifique. ${ }^{3}$ Mais s'il s'agit là d'une maladie, ils la considèrent comme «infantile». Cette orthodoxie malfaisante est inhérente aux situations de crise, comme le montre l'histoire de l'Europe occidentale, et elle s'effritera à mesure que disparaitront les dangers de contre-révolution et de guerre. ${ }^{4}$

Reste la question que pose le titre du livre dans ses première et deuxième éditions et à laquelle répond le titre de la troisième: le Communisme soviétique constitue-t-il une civilisation nouvelle? La réponse est affirmative et si catégorique que les Webb cesseront de formuler cette interrogation, d'ailleurs oratoire dès l'abord.

Mais quels sont les éléments principaux de cette civilisation nouvelle? Cinq facteurs importants se dégagent de l'étude fouillée consacrée à ce problème crucial.

Selon les Webb, le trait le plus significatif de la civilisation soviétique est l'instauration du planisme, ${ }^{5}$ le plan étant justement défini non seulement comme «un exercice de statistiques, mais aussi comme un instrument puissant ayant des effets dynamiques sur la volonté collective de la communauté». ${ }^{6}$ Ils scrutent très attentivement le système d'économie planifiée relevant ses mérites déterminants: il permet l'augmentation parallèle des investissements et du niveau de consommation; il supprime l'alternance de phases de «boom» et de dépression

1 Ibid., pp. 461-463.

2 Ibid., pp. $485-489$.

3 Ibid., pp. $801-807$, p. 970.

4 Ibid., p. 97^; Préface de B. Webb, p. XLVII

5 Soviet Communism, p. 495.

Ibid., p. 520. 
économique et par conséquent le chômage. Ils s'emploient aussi à réfuter la thèse selon laquelle le plan ne tiendrait pas compte de la liberté de choix des individus. ${ }^{1}$ Tout cela ne les empêche pas de mentionner certains échecs rencontrés par les Russes dans la réalisation de leurs premiers plans quinquennaux. ${ }^{2}$ En fait, selon les Webb, la planification soviétique aurait pu produire des résultats meilleurs encore, si elle ne s'était heurtée à des obstacles tels que l'inefficience d'une «couche intermédiaire de producteurs» (personnel d'employés et de comptables, contre-maitres et vérificateurs, machinistes des chemins de fer, magasiniers, etc....) et le sabotage souvent organisé. ${ }^{3}$

A côté du planisme qui conditionne l'ensemble de la vie de l'Union soviétique et modèle sa civilisation, il y a, comme conséquence proprement éthique et culturelle, l'élimination du mobile du profit. Les Webb $y$ insistent longuement sans s'attacher au fait - qu'ils signalent cependant à diverses reprises - que le mobile du profit continue à être pris en considération dans l'économie russe, bien qu'il ait cessé d'être la base fondamentale du régime économique soviétique. Les conséquences sociales et morales en sont considérables et, de l'avis des auteurs, extraordinairement bienfaisantes. ${ }^{4}$

Le troisième facteur de civilisation est l'existence d'une élite organisée, rassemblée dans le Parti communiste et les Komsomols, qui encadrent et guident l'ensemble de la nation dans les voies éclairées par l'idéologie communiste et les progrès de la science. ${ }^{5}$ Car, et c'est là un autre élément fondamental, la civilisation soviétique repose sur une religion nouvelle qui a remplacé les cultes abolis et décriés par celui de la Science. A ce phénomène, des fabiens, même assagis, ne pouvaient demeurer insensibles. Les Webb l'étudient tout au long du XIème chapitre de leur livre: "Le salut de l'Humanité par la Science» sans négliger ce qui leur parait être le revers de la médaille: l'athéisme militant et l'intolérance qui souvent l'accompagne. Mais ici encore il ne peut s'agir que d'un excès provisoire. ${ }^{6}$

Enfin, et comme une civilisation est aussi une éthique, il y a la nouvelle morale communiste qui s'exprime à travers une conception révolutionnaire du Bien et du Mal et qui bouleverse en Russie l'échelle des valeurs. Cette morale rassemble et proclame les vertus de dévouement au bien commun - de telle sorte que l'émulation entre

1 Ibid., p. 539, 541, 543.

2 Ibid., p. 565 .

3 Ibid., pp. 637, 642

${ }^{4}$ Ibid., pp. 651-652.

5 Ibid., pp. 900, 972.

B lbid., pp. 658, 746 .

7 lbid., pp. 807,815 . 
ouvriers n'exclut ni la coopération, ni la formation des moins habiles par les meilleurs ${ }^{1}$-, du désintéressement, de la sincérité ${ }^{2}$ et, last but not least, de la pureté des moeurs culminant en une forme nouvelle de puritanisme. $^{3}$

1 Ibid., p. 602.

${ }^{2}$ C.-à-d. de l'autocritique; ibid., pp. 630-1.

${ }^{3}$ Ibid., pp. $847-850$. 